\title{
Charge Localization in DNA Fibers
}

\author{
Francesco Luigi Gervasio, Alessandro Laio, and Michele Parrinello \\ Computational Science, Department of Chemistry and Applied Biosciences, ETH Zurich, USI Campus, Via Giuseppe Buffi 13, \\ CH-6900 Lugano, Switzerland \\ Mauro Boero \\ Institute of Physics, Special Nanoscience Project, University of Tsukuba, 1-1-1 Tennodai, Tsukuba, Ibaraki 305-8571, Japan
}

(Received 15 September 2004; published 21 April 2005)

\begin{abstract}
We study by first-principles molecular dynamics the mechanism of electron hole (positive charge) localization in a laboratory realizable radical cation $Z$ DNA crystal. We find that at room temperature structural deformation does not provide an efficient localization mechanism. Instead, we find evidence for the importance of changes in the protonation state for stabilizing the radical defect.
\end{abstract}

Charge transfer in DNA is currently the subject of intense theoretical and experimental investigation [1,2]. This is due both to a possible use of DNA as a component in nanoelectronic and electrochemical devices $[3,4]$ and to the fundamental role of conductivity in the oxidative damage of DNA [5,6]. Recent experiments have provided contradictory results, ranging from a highly conducting wire [7] and a proximity induced superconductor [8] to a semiconductor [9] or an insulator [10,11]. Such experiments are obviously technically complex since they require the handling of single molecules or small bundles of DNA, and the contact to the metallic leads and to the supporting surfaces can play a non-negligible role. Notwithstanding the experimental difficulties, a consensus has now been reached $[12,13]$. Wet DNA has been shown, by experiments on chemically modified or photosensitizer intercalated DNA [13], to be a charge carrier when its length is shorter than $\simeq 20 \AA$. While DNA helices longer than $\simeq 40 \AA$ or in dry conditions were generally found to be insulators or high-band-gap semiconductors [12]. These results are not completely surprising since DNA is a soft segmented molecule where randomness induced by distortion and defects crucially affects its conducting properties. Moreover, oxidation is expected to play a fundamental role as a parasitic event with respect to hole migration $[6,14]$. Two different mechanisms have been proposed to explain long-distance positive charge transport in duplex DNA: a coherent single step transport from donor to acceptor [15] or a hopping mechanism where the radical cation, initially trapped on one or several bases, moves to a different location when triggered [16]. The possibility of a longrange coherent single step transport can probably be ruled out by the disorder of DNA [17]. Hopping can, in principle, work via two mechanisms: tunneling when it is through only a few bases [18], or a phonon assisted polaronlike hopping model, where the introduction of a charge defect in DNA is accompanied by a structural change that stabilizes the defect [19]. In a recent review several theoretical and experimental arguments supporting a mechanism of charge transport by "large" polarons (i.e., a distortion accompanying the electron hole that is spread over a number of bases) were presented [20]. While the role of fluctuations in tuning DNA conductivity and a possible polaronlike hopping mechanism has been investigated in several experiments [19,21-24], our knowledge of the microscopic changes induced by the charge defect and its transfer is mostly based on indirect evidence. Three different stabilization mechanisms have been proposed: a change in the tilt angle of the bases [25], a change in the protonation state of $G$ [26-28], and a fluctuation in the position of counterions [29]. The first two mechanisms have been proposed mainly on the basis of the results of theoretical calculations. Indeed, theory is of great help in understanding these phenomena, but given the computational cost of full-scale calculations on realistic DNA systems, theoretical efforts to date have mostly been limited to small- and medium-sized model systems [30-32], to dry DNA molecules [10], or to larger systems using model Hamiltonian and semiempirical studies [25,33-38]. Model Hamiltonians are very useful in giving insight into physical mechanisms and sometimes can be in quantitative agreement with experiment, but they are somewhat restricted in their predictive value. Recent studies [29,39] have underlined the need to include the full complexity of the system and, in particular, the effect of solvation by water and counterions. In the present work, by performing first-principles calculations on a laboratory realizable system, we find direct evidence that in a $\operatorname{polyd}(G p C p)$ fiber the hole can be localized either by proton shift or by a change in the solvation shell of the counterions. Distortion of the helical parameters, on the other hand, is ineffective at room temperature.

We decided to study an oxidized crystalline continuous DNA fiber since we wish to simulate a laboratory realizable system that contains all the ingredients of an active DNA, but at the same time is much less complex than a disordered random sequence DNA dispersed in solution. It is for exactly the same reason that our system contains only 
$G$ and $C$. In recent experiments it was found that $\operatorname{poly}(d G)-$ poly $(d C)$ and poly $(d A)$-poly $(d T)$ exhibit different conduction mechanisms $[40,41]$. Inclusion at this stage of both $A: T$ and $G: C$ pairs would render the disentangling of different effects difficult. Moreover, in our system of choice the need to explore the long time scale fluctuations of DNA $[42,43]$ is dramatically reduced by the close packing and the greater rigidity of the system. Nonetheless, these are systems that have been characterized in the laboratory and are deemed useful in technological applications.

The system studied is a fully hydrated double strand self-complementary DNA [polyd $(G p C p)$ [44]; see Fig. 1(a)]. This is an infinitely repeated biopolymer, which in the unit cell contains 12 guanine:cytosine $(G: C)$ pairs in the $Z$ conformation. Polyd $(G p C p)$ adopts the $Z$ conformation only under conditions of high ionic strength and thus is rarely observed in nature. This choice, however, was made since in this particular crystal structure the DNA is continuous across crystal boundaries and because the $Z$ conformation exhibits less thermal fluctuations than either $A$ or $B$ forms [45]. The atomic and electronic structures of this system have already been thoroughly characterized via first-principles optimizations [39] while the oxidation of the guanine radical cation, a parasitic event with respect to hole migration, was studied with quantum mechanical (QM) or molecular mechanical (MM) calculations [6]. The simulation cell contains 12 base pairs, 654 heavy atoms and 540 hydrogen atoms (6 water molecules and $1 \mathrm{Na}+$ counterion per nucleotide). Periodic boundary conditions were used throughout. The calculations were per- a)

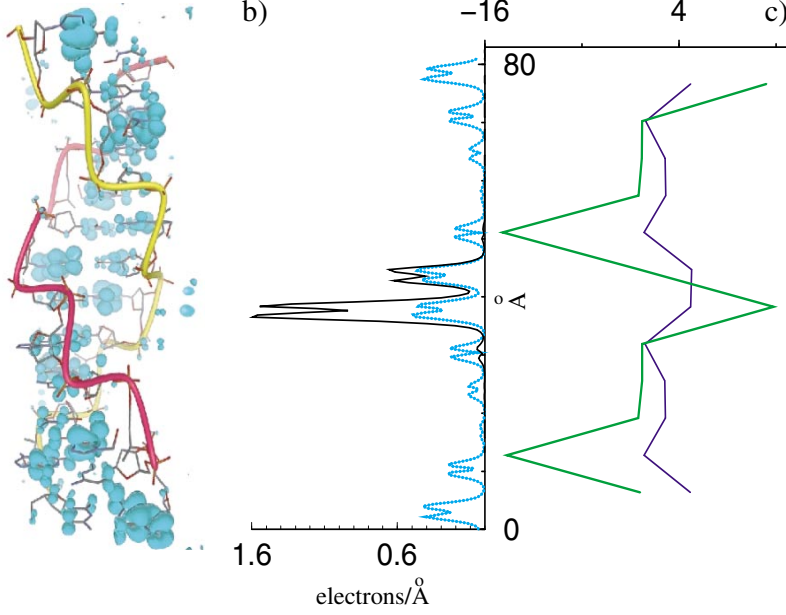

FIG. 1 (color). (a) View of the three-dimensional structure of the polyd $(G p C p)$ and of the spin density isosurface (in cyan) associated with the radical cation state at zero kelvin. The isosurfaces represented have a value of $10^{-4}$ electrons $\AA^{-4}$. (b) Projection of the electronic spin density $\rho_{s}(z)$ along the $z$ axis for the unit cell (cyan) and for the replicated cell (black). (c) Helical parameters of the structure in the local basis frame. The propeller twist is represented in blue, the tip in green. formed within the density functional theory (DFT) framework within the spin density generalization, as implemented in the CPMD code [46]. Gradient corrections after Becke-Lee-Yang-Parr [47,48] (BLYP) on the exchange and correlation functionals were used. The interaction between valence electrons and ionic cores was described by Martin-Troullier pseudopotentials [49]. The Kohn-Sham orbitals of the 3960 valence electrons were expanded in a plane wave basis set with an energy cutoff of 70 Ry. The Brillouin zone was sampled only at the $\Gamma$ point. All the Car-Parrinello molecular dynamics (MD) runs were performed at $300 \mathrm{~K}$ and the temperature was controlled via a Nosè-Hoover chain thermostat [50] on the ionic degrees of freedom. We used a fictitious electron mass $\mu=400$ and a time step of 2 a.u. The starting structures for the CarParrinello MD runs were obtained alternatively from the optimized structure of Ref. [39] or from three uncorrelated configurations obtained from a 4 ns classical MD run performed at $300 \mathrm{~K}$ with the Amber force field [51]. Local density functional theory has well known weaknesses in describing radical states. One of them is the incomplete cancellation of the self-interaction of the electron that leads to states which are too delocalized [52]. In spite of this fact, most of the states found here are localized and the extent of the localization is in good agreement with that found by a different method in Ref. [53]. Whenever we found delocalized states, we doubled the cell along the $x$ direction (perpendicular to the helical axis) and repeated the calculations using the BLYP functional as well as the recently developed Tao-Perdew-Staroverov-Scuseria functional [54], which is believed to better take into account the self-interaction corrections. Another important indicator for the quality of the theoretical treatment of an open-shell system like this is the expectation value of the total spin angular momentum, $\left\langle S^{2}\right\rangle$. Here it was calculated for all the relevant states following Wang et al. [55]. Helical parameters of the DNA were calculated with the 3DNA program that uses a base-centered reference frame [56,57].

Starting from the optimized geometry of Ref. [39] we removed one electron, performed a 2 ps molecular dynamics run at $300 \mathrm{~K}$, and then cooled the system to $0.4 \mathrm{~K}$ [58]. The residual disorder was very low as confirmed by the symmetry of the helical parameters [Fig. 1(c)]. In Figs. 1(a) and 1(b) (cyan) the localization of the charge density is shown. In the unit cell the calculated charge density is spread on 2 guanines and shows a periodicity $L / 2$ where $L$ is the length of the unit cell in the $z$ direction. However, repeating the calculations on the same geometry on a double cell, the $L / 2$ periodicity disappears, while the charge density on the two central guanines remains. The helical parameters related to the tilt of the bases follow the same pattern as $\rho_{s}(z)$ [Fig. 1(c), green and blue lines).

At higher temperatures the correlation between charge localization and structural distortion disappears, and there is no clear correlation between the charge localization and 
any of the helical parameters monitored. This is due to the disorder that breaks the symmetry between the different $G$. Already at $100 \mathrm{~K}$ the highest occupied molecular orbital (HOMO) is mainly localized on a particular $G$ plus residual nonzero contributions on several other bases [Figs. 2(a) and 2(b)]. During the $2 \mathrm{ps}$ run at $300 \mathrm{~K}$ we observed a similar behavior and the same is true for all the CarParrinello MD started from different classical MD snapshots, with the only difference that the peak in the spin density is found on different guanines. This finding is in good agreement with the observation of Schuster et al. of a large polaron state [19]. The calculated $\left\langle S^{2}\right\rangle$ during the dynamics remains close to 0.8 , very close to the correct value for a doublet (0.75), indicating that, in this case, the spin polarization is correctly described by DFT.

We now focus on one of the other two mechanisms proposed in the literature for the hole localization, namely, the fluctuation of the counterions and their solvation shell. During one exploratory Car-Parrinello MD at $300 \mathrm{~K}$, started from a geometry obtained from the classical MD trajectory, a critical fluctuation took place in which the solvation shell of a $\mathrm{Na}^{+}$opened up and the negative charge of the phosphate anion interacted with the positive $\mathrm{Na}^{+}$ charge without being screened by water. This fluctuation reduces the Coulomb repulsion and induces a charge localization on the closest sugar phosphate and the attached $G$. This finding is in agreement with the ion-gated charge hopping mechanism proposed by Landmann and Schuster who observed a counterion induced localization of the charge density very similar to the one that we observe on our system [29]. The spin density in this state shows strong oscillations close to the phosphate backbone and the value
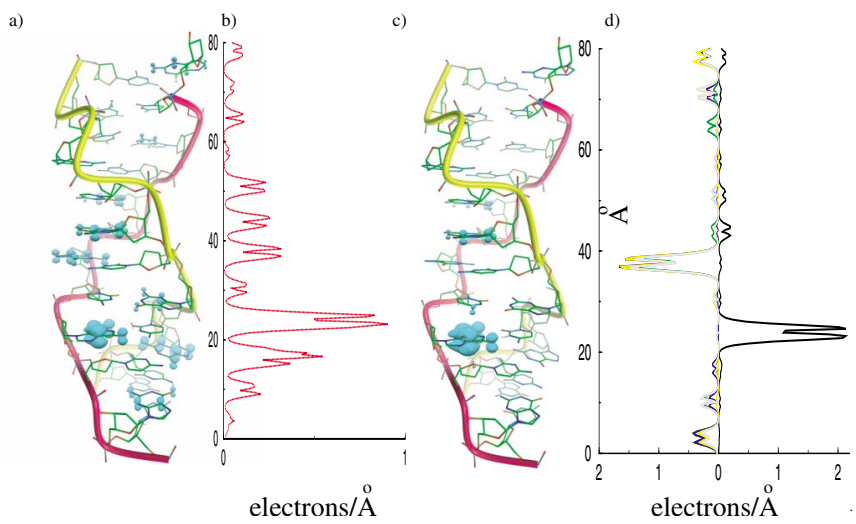

FIG. 2 (color). View of the structure of the polyd(GpCp) and of the spin density isosurface (in cyan) associated with the radical cation state (a) before and (c) after localization of the spin defect due to proton transfer from $G$ to $C$. The isosurfaces represented have a value of $10^{-3}$ electrons $\AA^{-3}$. (b),(d) Electronic spin density $\rho_{s}(z)$ projected along the $z$ axis. (b) The $\rho_{s}(z)$ corresponding to (a). (d) Right, the $\rho_{e}(z)$ corresponding to the status depicted in (c). (d) Left, four projections of the $\rho_{e}(z)$ corresponding to the shift of the proton from a different $G$ each taken every 48 fs. of $\left\langle S^{2}\right\rangle$ is 1.9 , rather different from the theoretical value of 0.75 . This might indicate that this state could be spuriously mixed with higher spin states and could be an artifact of DFT; therefore the present calculation cannot provide a conclusive answer concerning the possibility of an iongated charge hopping mechanism.

We then investigated the possibility of a proton-coupled charge transfer [27] by shifting a proton from the N1 of the $G$ to the N3 of the paired $C$. In fact, EPR experiments have shown that such a proton shift is possible [59]. We first displaced the proton to the base pair where the spin density has a maximum and then performed a geometry relaxation. We noticed a remarkable localization of the hole on $G$, leading to a situation in which the unpaired spin (the hole) is on $G$, yielding a $\left(G_{-H}\right)$ state, while $H^{+}$moves to $C$, which becomes protonated (Figs. 2(c) and 2(d), black lines). At variance with the previous case, the spin density shows no significant oscillations and the value of $\left\langle S^{2}\right\rangle$ was found to be 0.8 , very close to the expected 0.75 . The close relation between proton transfer and charge localization can be further demonstrated by repeating the calculation and moving the proton belonging to a different $G: C$ pair [Fig. 2(d), left]. In such a case, even if at the beginning the spin density on the newly chosen pair was small, a localization was induced on the site where the proton was displaced. From these calculations we can infer that hole transfer is coupled to proton transfer, since a positive charge is on the $C$ base and moves in a coupled way with the spin degrees of freedom localized in the partner $G$ base.

The decay of the spin localization is expected to be correlated with the difference in energy between the HOMO spin unpaired state and the HOMO-1 state. This is, indeed, the case since the HOMO-HOMO-1 gap goes from 0.01 to $0.05 \mathrm{eV}$ as the proton switches bases. We repeated the calculation after changing the protonation state of other $G: C$ couples and verified that in each case the charge becomes localized on the neutral $\left(G_{-H}\right)$.

On the basis of our calculations we can exclude that in polyd $(G p C p)$ charge localization is directly due to helix distortions. On the other hand, we find evidence for a proton-coupled charge transfer mechanism. This would be consistent with a wealth of experimental data on solvated DNA. Particularly striking are the data of H/D substitution and EPR. By replacing H with D Giese et al. [26] and Shafirovich et al. [27] have found a reduction of a factor of up to three in the charge mobility. They also found that the isotopic effect decreased with the increase of the length of the $A: T$ bridges. The EPR spectra, on the other hand, show clearly that in double helical $\mathrm{DNA}^{+} G$ undergoes deprotonation $[59,60]$. These experiments are difficult to explain without invoking a proton-coupled charge transfer as proposed in Ref. [27]. Also the decrease of the isotopic effect with increasing $A: T$ bridges is consistent with a coupled electron-proton transfer, since the proton shift cannot take place in $A: T$ pairs. Indeed, experiments 
show that $\operatorname{poly}(d G)-\operatorname{poly}(d C)$ and $\operatorname{poly}(d A)-\operatorname{poly}(d T)$ exhibit different conduction mechanisms [41].

We acknowledge a generous grant from the Earth Simulator Center (ES-JAMSTEC), Yokohama, which has made this calculation possible. M. B. is grateful to Takashi Ikeda for his valuable help. F. G. is grateful to L. Gilly for her valuable help.

[1] Longe-Range Charge Transfer in DNA I, Topics in Current Chemistry Vol. 236, edited by G. B. Schuster (SpringerVerlag, Heidelberg, 2004).

[2] Longe-Range Charge Transfer in DNA II, Topics in Current Chemistry Vol. 237, edited by G. B. Schuster (Springer-Verlag, Heidelberg, 2004).

[3] S. O. Kelley, N. M. Jackson, M. G. Hill, and J. K. Barton, Angew. Chem., Int. Ed. 38, 941 (1999).

[4] F. Lisdat, B. Ge, and F. W. Scheller, Electrochem. Comm. 1, 65 (1999).

[5] T. Douki et al., Top. Curr. Chem. 236, 1 (2004).

[6] F. L. Gervasio, A. Laio, M. Iannuzzi, and M. Parrinello, Chem. Eur. J. 10, 4846 (2004).

[7] R. Holmlin, P. Dandliker, and J. Barton, Angew. Chem., Int. Ed. Engl. 36, 2714 (1997).

[8] A. Y. Kasumov et al., Science 291, 280 (2001).

[9] D. Porath, A. Bezryadin, S. deVries, and C. Dekker, Nature (London) 403, 635 (2000).

[10] P. J. de Pablo et al., Phys. Rev. Lett. 85, 4992 (2000).

[11] C. Gomez-Navarro et al., Proc. Natl. Acad. Sci. U.S.A. 99, 8484 (2002).

[12] R. G. Endres, D. L. Cox, and R. R. P. Singh, Rev. Mod. Phys. 76, 195 (2004).

[13] D. Porath, G. Cuniberti, and R. Di Felice, Top. Curr. Chem. 237, 183 (2004).

[14] B. Giese and M. Spichty, Chem. Phys. Chem. 1, 195 (2000).

[15] C. J. Murphy et al., Science 262, 1025 (1993).

[16] D. Ly, Y. Kan, B. Armitage, and G. B. Schuster, J. Am. Chem. Soc. 118, 8747 (1996).

[17] A. Troisi and G. Orlandi, J. Phys. Chem. B 106, 2093 (2002).

[18] J. Jortner, M. Bixon, A. A. Voityuk, and N. Rosch, J. Phys. Chem. A 106, 7599 (2002).

[19] G. B. Schuster, Acc. Chem. Res. 33, 253 (2000).

[20] E. Conwell, Top. Curr. Chem. 237, 73 (2004).

[21] P. T. Henderson et al., Proc. Natl. Acad. Sci. U.S.A. 96, 8353 (1999).

[22] D. Ly, L. Sanii, and G. B. Schuster, J. Am. Chem. Soc. 121, 9400 (1999).

[23] M. A. O’Neill et al., Angew. Chem., Int. Ed. 42, 5896 (2003).

[24] C. Wan et al., Proc. Natl. Acad. Sci. U.S.A. 96, 6014 (1999).

[25] R. Bruinsma, G. Grüner, M. R. D’Orsogna, and J. Rudnik, Phys. Rev. Lett. 85, 4393 (2000).

[26] B. Giese and S. Wessely, Chem. Commun. 20, 2108 (2001).

[27] V. Shafirovich, A. Dourandin, and N.E. Geacintov, J. Phys. Chem. B 105, 8431 (2001).
[28] S. Steenken, Biol. Chem. 378, 1293 (1997).

[29] R. N. Barnett et al., Science 294, 567 (2001).

[30] J. Reynisson and S. Steenken, Phys. Chem. Chem. Phys. 4, 527 (2002).

[31] H. Sugiyama and I. Saito, J. Am. Chem. Soc. 118, 7063 (1996).

[32] Y. Yoshioka et al., J. Am. Chem. Soc. 121, 8712 (1999).

[33] G. Brunaud, F. Castet, A. Fritsch, and L. Ducasse, Phys. Chem. Chem. Phys. 5, 2104 (2003).

[34] F. C. Grozema, L. D. A. Siebbeles, Y. A. Berlin, and M. A. Ratner, Chem. Phys. Chem. 3, 536 (2002).

[35] I. V. Kurnikov, G. S. M. Tong, M. Madrid, and D. Beratan, J. Phys. Chem. B 106, 7 (2002).

[36] S. Roche, Phys. Rev. Lett. 91, 108101 (2003).

[37] S. Roche, D. Bicout, E. Maciá, and E. Kats, Phys. Rev. Lett. 91, 228101 (2003).

[38] A. A. Voityuk, K. Siriwong, and N. Rösch, Phys. Chem. Chem. Phys. 3, 5421 (2001).

[39] F. L. Gervasio, P. Carloni, and M. Parrinello, Phys. Rev. Lett. 89, 108102 (2002).

[40] B. Giese et al., Nature (London) 412, 318 (2001).

[41] K.-H. Yoo et al., Phys. Rev. Lett. 87, 198102 (2001).

[42] D. L. Beveridge and K. J. McConnell, Curr. Opin. Struct. Biol. 10, 182 (2000).

[43] E. B. Brauns et al., J. Am. Chem. Soc. 121, 11644 (1999).

[44] C. Ban, B. Ramakrishnan, and M. Sundaralingam, Biophys. J. 71, 1215 (1996).

[45] K. K. Irikura, B. Tidor, B. R. Brooks, and M. Karplus, Science 229, 571 (1985).

[46] CPMD V3.5, Copyright IBM Corp, 1990-2001, Copyright MPI fuer Festkoerperforschung, Stuttgart, 1997-2001).

[47] A. D. Becke, Phys. Rev. A 38, 3098 (1988).

[48] C. Lee, W. Yang, and R. G. Parr, Phys. Rev. B 37, 785 (1988).

[49] N. Troullier and J.L. Martins, Phys. Rev. B 43, 1993 (1991).

[50] G. Martyna, M. Klein, and M. Tuckerman, J. Chem. Phys. 97, 2635 (1992).

[51] W. D. Cornell et al., J. Am. Chem. Soc. 117, 5179 (1995).

[52] J. Laegsgaard and K. Stokbro, Phys. Rev. Lett. 86, 2834 (2001).

[53] J. P. Lewis et al., J. Phys. Chem. B 107, 2581 (2003).

[54] J. Tao, J. P. Perdew, V. N. Staroverov, and G. E. Scuseria, Phys. Rev. Lett. 91, 146401 (2003).

[55] J. Wang, A. D. Becke, and V. H. Smith, Jr., J. Chem. Phys. 102, 3477 (1995).

[56] The calculated helical parameters include shear, stretch, stagger, buckle, propeller twist, opening and $x$ displacement, $y$ displacement, helical rise, inclination, tip, and helical twist.

[57] X. J. Lu and W. K. Olson, Nucleic Acids Res. 31, 5108 (2003).

[58] Starting from $300 \mathrm{~K}$ the temperature was cooled to less than $1 \mathrm{~K}$, rescaling the velocities by a factor of 0.99 each step.

[59] K. Hildebrand and D. Schulte-Frohlinde, Free Radic. Res. Commun. 11, 195 (1990).

[60] O. Schiemann, N. J. Turro, and J.K. Barton, J. Phys. Chem. B 104, 7214 (2000). 